Revista de Psicología de la PUCP. Vol. XV, 2, 1997.

\title{
EL ESTILO PSICOLÓGICO COMO ESTUDIO DE LA DIVERSIDAD HUMANA: UN EJEMPLO BASADO EN LOS ESTILOS DE VIDA
}

\author{
Ma Pilar Sánchez-López ${ }^{1}$ \\ Universidad Complutense de Madrid España
}

Este estudio considera el Estilo Psicológico (EP) como categoría supraordinal en la organización de la personalidad y como base del enfoque del cambio. El estilo organiza la conducta, tiene un carácter eminentemente global, es imprescindible para el mantenimiento adaptativo del individuo y puede considerarse como un conjunto de pautas de interpretación, donación de significados y respuestas. Dado su carácter globalizador, el EP es aplicable a muchos campos. Se presenta un trabajo empírico sobre estilos de vida (EV), proponiéndose su operativización mediante la distribución del tiempo. Se presenta el instrumento original creado (ADIT). Los datos indicaron que podemos utilizar la distribución del tiempo como operativización del EV, puesto que los perfiles encontrados tienen un claro sentido psicológico y las relaciones con las variables demográficas tienen también sentido psicológico.

Palabras clave: estilo psicológico, diferencias individuales, estilos de vida, distribución del tiempo.

Psychological style as a way to study human diversity: An example based on life styles This paper considers Psychological Style (PS) as a supraordinal category in the personality organization and as an approach of change. The PS organizes behavior, has an eminet global character, is essential for interpretation, and could be considered as a set of guidelines. Given its encompassing nature, PS is useful in many fields. An empirical research on Life Styles (LS) is presented, in which is proposed its operativization through time distribution. An original instrument on time distribution is given (ADIT). The data collected indicated that time distribution can be used as an operativisation of LS, since the profiles found have a clear psychological significance and the relation with demographic variables also have a psychological meaning.

Keywords: psychological style, individual differences, life styles, time distribution.

1. Catedrática de Psicología Diferencial. Directora del Departamento de Psicología Diferencial y Psicología del Trabajo. E-mail: psdif02@sis.ucm.es. 



\section{El concepto de estilo psicológico}

La finalidad de la primera parte de este trabajo es proponer el concepto de estilo psicológico como categoría de clasificación para estudiar la diversidad humana. No se pretende sustituir radicalmente las categorías de clasificación clásicas, como rasgo, tipo o factor; siguen siendo útiles y válidas para el estudio de la diversidad. Sin embargo, el concepto de estilo psicológico (EP) tiene algunas ventajas sobre todo si nos interesa una perspectiva más dinámica que estática del comportamiento humano, y sí queremos, parafraseando a Carlson, 1971 "encontrat al individuo" cuando estudiamos la personalidad.

Partimos de la consideración del EP:

1. Como categoría supraordinal en la organización de la personalidad

2. Como base del enfoque del cambio

En primer lugar, la consideración del EP como categoría supraordinal parte de unos supuestos específicos que, en resumen, podríamos enunciar de la forma siguiente:

La consideración de la personalidad como un conjunto organizado y jerarquizado, en cuya cima estaría el EP. Dentro de la tradición de Royce y Powell (1983), se definiría como un sistema que proporciona la dirección y el enfoque a los sistemas de nivel más bajo, participando e integrando los dos grandes subsistemas de diferenciación interindividual, el sistema cognitivo y el afectivo-motivacional (ver Sánchez-Canóvas y SánchezLópez, 1994) porque "recluta" las aptitudes y los rasgos afectivos implicados cuando un sujeto actúa en una situación determinada. 
Aunque nuestra concepción no coincide totalmente con la mantenida por Royce y Powell, es bueno recordar en este planteamiento general, el Modelo Sistemático de Personalidad Integradora (Figura 1) defendido por estos autores (ver Royce y Powell, 1983).

El sistema de estilos forma junto con el de valores, el nivel integrativo de la personalidad. Son los dos sistemas que proporcionan la dirección y el enfoque de la atención que requieren los sistemas de bajo nivel, el sensorial y el motor en el primer nivel, la cognición y el afecto en el siguiente. Tanto los estilos como los valores proporcionan relaciones conceptuales entre la cognición y el afecto, y, en este sentido, la definición de estilos es semejante a la de Reuchlin (1990) que veremos más adelante y a su insistencia en la integración entre el sistema cognitivo y el conativo. Lo que, dentro del modelo de Royce y Powell, singulariza el sistema de estilos (frente al de valores) es que la misión del primero es seleccionar los modos de procesamiento cognitivo y afectivo para poder dirigir el enfoque de la atención.

Con respecto al segundo punto, el EP como base del enfoque de cambio, nuestra postura podrá resumirse diciendo que si el objeto de la psicología es el estudio del individuo en su contexto y si, al interesarnos en el estudio de la diversidad humana, nos interesa la diversidad de los individuos en la diversidad de contextos, entonces nos interesa conocer no sólo la estructura, sino también la dinámica y el desarrollo de las diferencias individuales. Esto es, nos interesa estudiar el patrón de cambio, que es lo que, en muchas ocasiones caracteriza realmente al sujeto. No la "foto fija", que puede ser engañosa, sino la "imagen en movimiento". Brevemente, se propone que para estudiar ese patrón de cambio (al que nos referimos un poco más adelante), es más útil, en muchas ocasiones, utilizar como categoría de clasificación de la realidad (en nuestro caso, de la diversidad humana) la categoría de estilo psicológico, que tiene ventajas sobre las de tipo, rasgo o factor (aunque no se pretende desterrar estas últimas), en el sentido de que nos permite más fácilmente encontrar la regularidad de ese cambio. Y ello es así porque el EP nos permite reconocer lo "invariante" de una persona a través de conductas muy distintas, es decir, nos permite reco- 


\section{Figura 1}

Representación gráfica de la Teoria Multifactorial Sistemática de Royce y Powell (1983). El sombreado es nuestro

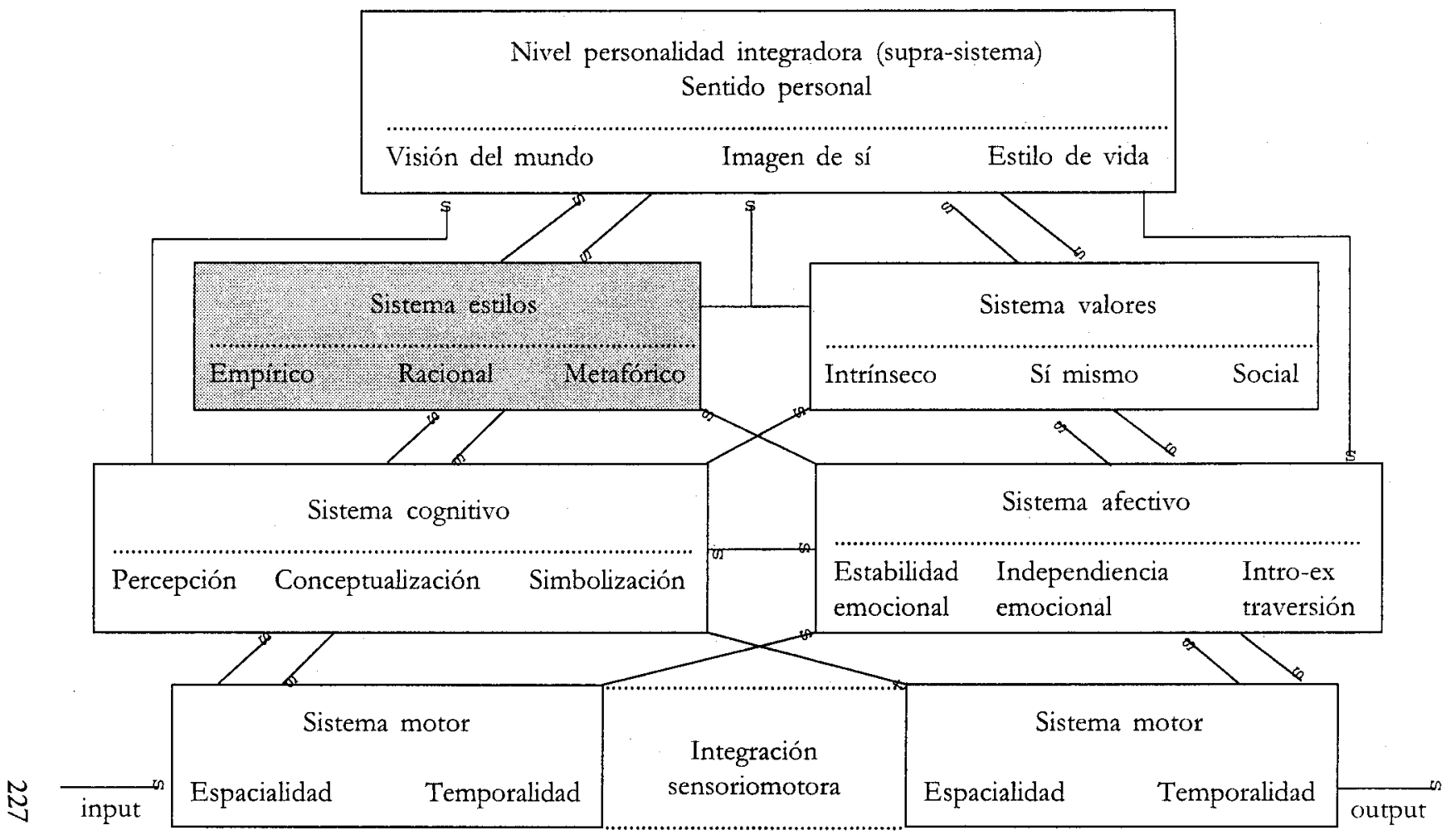


nocer al individuo. Veamos el ejemplo de un tipo de estilo muy conocido, los estilos cognitivos, $y$, dentro de ellos, el más conocido probablemente, el de dependencia-independencia de campo (DC-IC), propuesto y estudiado por Witkin y su equipo (Quiroga y Sánchez-López, 1987; Witkin, 1949), reconocemos al sujeto dependiente de campo a través de conductas tan dispares como una interacción social o una tarea perceptiva, para poner ejemplo de contextos claramente distintos.

A partir de todo lo dicho, se propone una definición del EP como un conjunto de modalidades de funcionamiento psicológico observables, que:

- Están en la cúspide el Sistema jerárquico que constituye la personalidad,

- Organizan e integran el resto de los niveles,

- Traspasan los dos grandes sistemas de diferenciación psicológica, el cognitivo y el afectivo-motivacional,

- Hacen referencia a diferencias más cualitativas que cuantitativas,

- Se expresan mediante dimensiones más que mediante categorías discontinuas $y$

- Proporcionan unidad y coherencia al comportamiento de un sujeto y/o de un grupo de sujetos.

\section{El patrón de cambio y el estilo psicológico}

La insistencia que estamos haciendo en el patrón de cambio pone de manifiesto que el marco de referencia general en el que se inscribe esta conceptualización de EP concede especial importancia a los aspectos procesuales de la personalidad. La idea básica es que los procesos de personalidad son fenómenos relativamente estables, pero no estáticos, es decir, son patrones consistentes de cambio. Son lo que, en terminología de Larsen (1989), se denominan consistencias de segundo orden. En resumen, estas consistencias de segundo orden son: 
- Regularidades o patrones temporales

- Identificación a través del análisis del individuo

- Que son útiles para describir diferencias fiables entre distintos individuos

- Mediante una aproximación al estudio de la personalidad que comienza por el análisis del individuo (buscando patrones temporales) para llegar al nivel de grupo (buscando diferencias significativas entre sujetos).

- Utilizando, por lo tanto, el paradigma idiográfico-nomotético propuesto por Epstein (1983) o el enfoque centrado en el individuo, (Krahé, 1990).

Podemos ver la significación de las consistencias de segundo orden a través de dos ejemplos. Por una parte, el propio concepto de trastorno maniaco-depresivo una "consistencia no estática": el sujeto maníaco-depresivo muestra un cambio constante, cuyo carácter cíclico es, precisamente, lo que tiene consistencia. Por otra, los humores o estados de ánimo, que, por su propia naturaleza, pertenecen a la parte menos estable de la personalidad: asumimos que las personas tratan de regular sus estados de ánimo de tal forma que ayuden al mantenimiento de estados afectivos positivos y eviten los negativos. Si, cuando intentamos comprenderlos, utilizamos la evaluación día a día, es posible que lo que surja tenga una apariencia caótica. Pero si utilizamos un modelo de análisis que incorpore un marco temporal más amplio, seguramente aparecerán, en ese aparente caos, patrones consistentes en la regulación del humor, patrones con una finalidad, inteligentes y predecibles. Es decir, aparecerá, por un lado, una "estabilidad no estática" en los esfuerzos que el individuo hace para mantener el equilibrio emocional. Y, por otro lado, es bastante probable que aparezcan también diferencias interindividuales e intergrupales en esos patrones consistentes. 


\section{Los campos de aplicación de los estilos psicológicos}

Como resumen tenemos que, el EP:

\section{Da unidad al cambio}

La categoría de clasificación "estilos psicológicos" responde mejor que otras a la finalidad de estudiar "las regularidades a través de las irregularidades", es decir, las regularidades que se pueden entresacar a partir del cambio. No parece descabellado proponer que lo que nos sirve para reconocer la unidad de la conducta a través de sus cambios, lo que nos permite reconocer que es un mismo individuo el que se comporta (aún a través de manifestaciones distintas) es el "estilo". En este sentido, acierta el lenguaje popular cuando habla de que "reconocemos" el estilo de tal o cual persona, que haberse comportado así, es "su estilo".

Asimismo, en la misma línea, el EP sirve mejor al concepto de coherencia, sobre todo como la entienden Buss y Cantor (1989), cuando hablan de las nuevas formas de coherencia de la personalidad. La coherencia, como el estilo, es considerada como una característica dinámica, no estática, de la personalidad, y, en ambos casos, la variable "tiempo" tiene una importancia fundamental.

\section{Sirve de integración entre las variables cognitivas y las conativas}

Toda la corriente de new look insiste, es verdad, en esa integración. A estas alturas, no hace falta insistir más en su necesidad. Dentro de esta corriente, los trabajos de Witkin (1949) sobre dependencia-independencia de campo son la base de la aparición del término "estilo cognitivo", que ha propiciado tantos trabajos interesantes (Quiroga, 1994; Quiroga y Sánchez-López, 1987). Es cierto que, desde el primer momento, el estilo cognitivo se plantea como un intento de integración entre el sistema cognitivo y el sistema afectivo- motivacional, artificial y excesivamente separados, al menos en la práctica de la investigación. Pero no es menos cierto que al conservar el término "cognitivo" en su denominación, puede incitar a centrarse en un solo aspecto, el cognitivo. 
Esto es lo que hace que Reuchlin (1990) proponga el término "estilos de comportamiento", conceptualizándolo como variables latentes que se postulan como causa de las correlaciones entre variables, pero que al pertenecer tanto al dominio cognitivo como al conativo se diferencian tanto de las aptitudes por una parte como de las variables clásicas de personalidad por otra.

Las diferencias con los rasgos de personalidad son más difíciles de establecer que con las aptitudes. Los rasgos de personalidad utilizados sobre todo por Eysenck y Catell son a menudo bipolares, como los estilos, y algunos de ellos son definidos como esquemas directores de la conducta, e implican a la vez (como los estilos) estructuras cognitivas y conativas, pero Reuchlin propone algunas deferencias de matiz entre las variables clásicas de personalidad y los estilos, que podrían resumirse de la forma siguiente:

- La personalidad está constituida por un conjunto de dimensiones más elementales, los rasgos, que son los que se investigan. Por el contrario, cada estilo constituye una sola dimensión sobre la que cada individuo ocupa teóricamente una posición determinada. El estilo es una causa única que puede tener manifestaciones diferentes, los rasgos son una pluralidad de componentes en los que el conjunto, la suma de ellos es la causa fundamental de las conductas observadas. Sin embargo, es cierto que en determinados rasgos de personalidad, como la introversión-extraversión de Eysenck, esta distinción tiende a difuminarse $y$, de hecho, este rasgo se considera a veces como un estilo.

- Con respecto a la noción de tipo, la diferencia fundamental radica en que muy a menudo, la noción de tipo es esencialmente descriptiva, mientras que los estilos tienen en general la ambición de explicar los parecidos observados en la conducta de individuos diferentes. Los tipos, además, suelen ser discontinuos, mientras que los estilos pretenden ser dimensiones continuas.

- Tanto los rasgos como los tipos, al menos como son utilizados en la actualidad, sólo cubren generalmente un espectro de conductas relativamente restringido. Por el contrario, se pretende que los estilos 
se refieran a una abanico de conductas mucho más amplio, y, desde luego, que incluya los aspectos cognitivos y conativos de la conducta.

\section{Organiza y dirige la conducta}

Todas las definiciones dadas por los diversos autores coinciden en señalar este aspecto, desde las de Royce y Powell (1983), señalándolo como sistema organizador e integrador de la personalidad (junto con el sistema de valores), pasando por la concepción de "regla de regla" del sistema adleriano (Shulman y Mosak, 1988), hasta su concepción como algo imprescindible para el mantenimiento adaptativo del individuo (Ibañez, 1990) o la definición como conjunto de pautas de interpretación, donación de significados y respuestas (Marina, 1993). En una concepción sistémica de la personalidad, cuyo paradigma podría ser el modelo de personalidad integradora defendido por Royce y Powell, cuanto más cerca de la cima está un sistema, mayor es su papel integrador de la personalidad. Es comprensible, por tanto, dado lo expuesto hasta ahora, que los estilos tengan un papel importante en esa función de integración y dirección.

\section{Su versatilidad facilita su uso en un amplio espectro de campo de aplicación}

Efectivamente, desde los campos de aplicación más generales hasta los más específicos, su versatilidad ha hecho que el término se haya aplicado a un amplio espectro de campos relacionados con la psicología. Las referencias que con más frecuencia aparecen en la bibliografía podrían resumirse de la forma siguiente:

- Estilos cognitivos.- Desde los primeros trabajos de Witkin y sus colaboradores, que dieron lugar a la propuesta del más conocido, la DCIC, los distintos tipos de estilos cognitivos propuestos han gozado de una gran popularidad, aunque también han recibido importantes críticas, sobre todo desde el punto de vista conceptual (para una revisión, Quiroga, 1994). Entre los más conocidos, los de amplitud de categorización, complejidad cognitiva, toma de riesgos us. cautela, adaptadores-innovadores y reflexividad-impulsividad. 
- Estilos de aprendizaje.- La diferencia con los estilos estilos cognitivos no es a veces muy clara. Los primeros suelen ser mucho más generales, es cierto, mientras que los estilos de aprendizaje se refieren exclusivamente a las preferencias personales en el aprendizaje de la información (Furnham, 1995). Los trabajos más conocidos son los de Kolb (1984).

- Estilos de desarrollo- La denominación ha sido propuesta por Reuchlin (1990), e incluye los trabajos y los datos sobre diferencias en el desarrollo de la conducta exploratoria, el lenguaje y la inteligencia. En cada uno de estos apartados existen datos empíricos y tendencias de investigación que pueden ser el embrión de un cuerpo importante de conocimiento que complete el estudio de los estilos.

- Estilos de vida.- Entendidos como la parte más visible y externa de los estilos, lo que no quiere decir que sea la menos importante. De hecho, los estilos de vida se relacionan empíricamente con otras variables psicológicas, desde las consideradas tradicionalmente diferenciales, como edad, sexo, clase social, hasta cualquier otra variable o conjunto de variables que sirvan para caracterizar grupos de sujeto. El término "estilos de vida" aparece en las ciencias sociales de la mano de Max Weber, en el sentido de modo social de vivir: tiene una característica cultural, es típico de un determinado pueblo o sociedad. En parte de la bibliografía actual sobre los estilos de vida se conserva esta significación, por ejemplo, cuando se estudia un grupo específico y típico como veremos en el último de estos apartados. Por otra parte, el término es emblemático de la psicología adleriana. Dentro de esta corriente, se define como el patrón de reglas, la "regla de reglas", el patrón organizativo supraordenado que dirige la conducta (Shulman y Mosak, 1988). En los puntos siguientes haremos referencias a algunas aplicaciones de estos estilos de vida.

- Estilos de enfermar (o Estilos de vida y salud).- Desde sus comienzos, tanto el término "estilo de vida" como el afin de "hábitos de vida" aparecieron relacionados con el tema de la enfermedad. La predisposición a determinados tipos de enfermedades, desde la teoría psicoanalítica, el intento de establecer si son o no las variables de personalidad las que llevan a 
determinados hábitos de vida que impliquen mayor riesgo a contraer determinadas enfermedades o la comprobación de que determinadas estrategias de afrontamiento pueden mejorar las tasas de supervivencia y acortar el período de enfermedad son ejemplos de esta unión. Quizá los trabajos más conocidos se refieren a las relaciones encontradas entre extraversión y los hábitos de fumar; en los distintos trabajos de Eysenck, y con la personalidad tipo $\mathrm{A}$ y las enfermedades cardiovasculares (Contrada, Wright y Glass, 1985).

- Estilos de consumo.- La aplicación de los estilos de vida al estudio del consumo es una de las áreas con mayor tradición y mayor número de trabajos empíricos. En realidad, el interés por el estudio de los estilos de vida en la población norteamericana tiene su embrión en un primer trabajo del autor de la tipología de los VALS (Values and Lifestyles), Mitchel, sobre los estilos de consumo (Cooper, Stern y Mitchel, 1960).

- Estilos de vida y grupos.- Caracterizados por las variables de diferenciación clásicas, como edad, sexo, clase social, etc. En este contexto se utiliza el concepto más weberiano de estilo de vida, en el sentido de que caracteriza a un grupo social determinado. Los trabajos de Hendry, Shuksmith, Love y Geendinning (1993) con adolescentes, de Scase y Goffe (1989) con los ejecutivos o de Sekaran (1985) con parejas de doble carrera sería representativos de este grupo.

En resumen, y para finalizar este apartado, la concepción que propugnamos en este trabajo podría representarse gráficamente en la Figura 2.

\section{Estilos de Vida y Satisfacción Vital}

En este último apartado se presenta un ejemplo de cómo puede trabajarse empíricamente con los EP y, en concreto, con una de sus aplicaciones, los estilos de vida. El estilo de vida (EV) puede definirse como el conjunto de soluciones estratégicas que adopta el individuo para poder cumplir sus planes y objetivos globales; en la práctica, adoptan la forma 


\section{Figura 2}

Resumen de la propuesta sobre estilos psicológicos

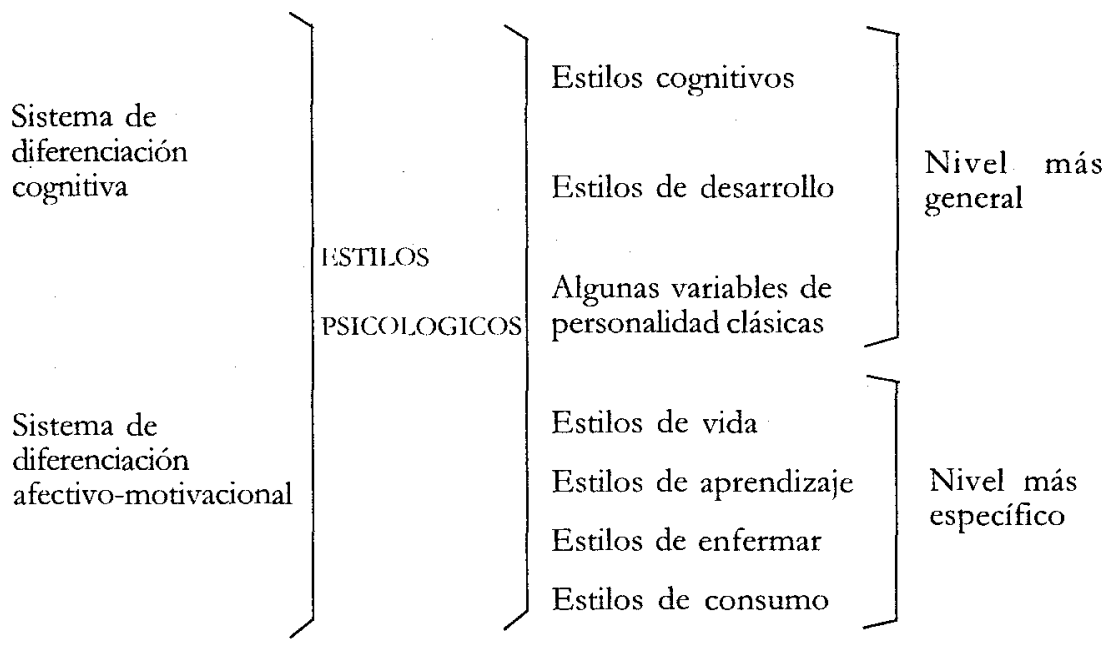

de patrones de vida episódicos (Shulman y Mosak, 1988; Ibañez, 1990). Se relacionan empíricamente con otras variables psicológicas, desde las consideradas tradicionalmente diferenciales, como sexo, edad o clase social, hasta variables como satisfacción y bienestar psicológico (Argyle 1987; Strack, Argyle y Schwarz, 1991); en aspectos más específicos, el trabajo de Larson, Richards, Raffaelli, Ham y Lewell (1990) se centra en el EV en relación con las satisfacción en la infancia y la adolescencia; Sánchez-Canovas (1993) relaciona factores de riesgo y estrés con el bienestar psicológico; Barling (1990) analiza el estilo de vida producido por las relaciones entre trabajo y familia; Leslie y Anderson (1988) y Cooper, Chasin, Braver, Zeis y Khavari (1986), el estilo de vida de la pareja y la satisfacción marital; y más específicamente, un EV concreto, el de las parejas de doble carrera con la felicidad aparece en los trabajos de Yogev (1982), Gilbert (1985), Sekaran (1985) y Radmika y Prakash (1987).

Un problema fundamental es cómo se operativiza el EV. En la bibliografia aparece algunas propuestas, aunque a veces sean más implícitas que 
explícitas. La propuesta de este trabajo es que la operativización puede hacerse adecuadamente a través de la distribución del tiempo, sobre la base tanto de la racionalidad teórica (importancia del tiempo en la vida humana, McGrath, 1988, como revisión) como en los datos empíricos (Campbell, Converse y Rodgers, 1976; Argyle, 1987; Durán, 1987; Izquièrdo del Río y Rodríguez 1988). En concreto, se propone operativizar el EV mediante la distribución del tiempo de los sujetos a lo largo de las 24 horas del dia, durante una semana estándar de su vida. Para ello, se ha construido un instrumento original, al que nos referiremos más adelante.

En breve, el objetivo fundamental del trabajo empírico que presentamos es demostrar que los EV pueden operativizarse mediante la distribución del tiempo que realizan empíricamente los sujetos, medida a través de un instrumento original, el Autoregistro de Distribución del Tiempo (ADIT).

Para demostrarlo, deberemos encontrar que los sujetos muestran perfiles diferenciales de distribución del tiempo, con caracterización psicológica, y que estos perfiles se relacionan con las variables demográficas clave. Por resultados encontrados en nuestros trabajos anteriores (Quiroga y Sánchez, 1997; Sánchez y Quiroga, 1995) se escogen como variables demográficas importantes el sexo, el tipo de pareja y el ciclo vital. De ellas, la única variable que tiene que ser definida teóricamente y operativizada es el "tipo de pareja".

Con respecto a su definición, hemos utilizado la distinción entre tipos de pareja, de acuerdo con la bibliografía existente: las de doble carrera, de doble ingreso y tradicionales. Las familias de doble carrera (término acuñado por Rapaport y Rapaport, 1969) son una variación de la familia nuclear, en la que ambos esposos persiguen una carrera profesional ininterrumpida y también establecen y desarrollan una vida familiar que a menudo incluye hijos. Son un grupo de tamaño creciente en nuestra sociedad, con un estilo de vida propio y que, a pesar de que su número es relativamente escaso (aunque creciente), constituyen un segmento de la población que puede considerarse como prototipo, como marco de referencia para segmentos 
poblacionales más amplios. La distinción con las parejas de doble ingreso radica en su acercamiento a la situación laboral; en estas últimas, uno o ambos esposos tienen trabajos que, aunque sean carreras, en el sentido académico, no requieren un alto grado de compromiso individual con el rol laboral o una constante actualización del conocimiento profesional. En las familias tradicionales sólo uno de los miembros aporte el dinero, mientras que el otro, normalmente la mujer, es el ama de casa.

Según su definición a partir de la bibliografía, cada uno de los tres tipos se operativizan mediante la actividad laboral de ambos miembros de la pareja, completada por algunos de los datos demográficos (tipo de contrato, número de horas de trabajo a la semana, etc.). Con respecto a la variable que se ha denominado "ciclo vital", hay que señalar que está constituida por la conjunción de "tener/no tener hijos" y "edad de los participantes". Los análisis estadísticos se realizan con cada una de ellas por separad, pero dado que sus tendencias de relación con otras variables van siempre en la misma dirección, frecuentemente nos referimos a ella como "ciclo vital".

En consecuencia, se formula las siguientes hipótesis:

1. La distribución del tiempo, durante los días laborales de la semana y durante el fin de semana, permite encontrar perfiles de sujetos, con significación psicológica.

2. Estos perfiles de distribución de tiempo mostrarán relaciones con variables demográficas como sexo, tipo de pareja y ciclo vital.

\section{Metodologia}

\section{Participantes}

Se trabajó con 74 personas emparejadas (después de la mortandad experimental), pertenecientes a los tres tipos de parejas señalados, y cuya descripción demográfica se presenta en el Cuadro 1. 


\section{Cuadro 1}

Resumen general de las caracteristicas demográficas del grupo analizado

\begin{tabular}{|c|c|c|}
\hline Caracteristicas. & Distribucion & 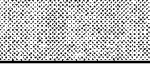 \\
\hline \multirow[t]{3}{*}{ - Tipo de pareja } & Tradicionales: 24 & $27.3 \%$ \\
\hline & Doble carrera: 30 & $34.1 \%$ \\
\hline & Doble ingreso: 34 & $38.6 \%$ \\
\hline \multirow{5}{*}{ - Estudios } & Primarios: 24 & $8.0 \%$ \\
\hline & Básica: 5 & $5.7 \%$ \\
\hline & Bachiller, FP: 17 & $19.3 \%$ \\
\hline & Título Grado medio: 8 & $9.1 \%$ \\
\hline & Título Superior: 51 & $58.0 \%$ \\
\hline \multirow[t]{8}{*}{ - Trabajo actual } & Amas de casa: 12 & $13.6 \%$ \\
\hline & No especializado: 1 & $1.3 \%$ \\
\hline & Especializados: 7 & $9.2 \%$ \\
\hline & Administrat., maestros: 19 & $25.0 \%$ \\
\hline & Peq. comerciante, mandos & \\
\hline & intermedios: 7 & $9.2 \%$ \\
\hline & Profesiones liberales: 36 & $47.4 \%$ \\
\hline & Empresarios directivos: 7 & $7.9 \%$ \\
\hline \multirow[t]{4}{*}{ - Contrato actual } & Sin trabajo: 12 & $13.6 \%$ \\
\hline & Temporal: 15 & $19.6 \%$ \\
\hline & Indefinido: 56 & $73.7 \%$ \\
\hline & Autónomo: 5 & $6.6 \%$ \\
\hline \multirow[t]{6}{*}{ - Horario laboral } & Sin trabajo: 12 & $13.6 \%$ \\
\hline & Menos de $20 \mathrm{~h} / \mathrm{s}: 2$ & $2.6 \%$ \\
\hline & Entre $21-34 \mathrm{~h} / \mathrm{s}: 10$ & $13.2 \%$ \\
\hline & Entre $35-40 \mathrm{~h} / \mathrm{s}: 38$ & $50.0 \%$ \\
\hline & Entre $41-60 \mathrm{~h} / \mathrm{s}: 26$ & $34.2 \%$ \\
\hline & Más de $60 \mathrm{~h} / \mathrm{s}$ : & - \\
\hline \multirow[t]{4}{*}{ - Número de hijos } & Sin hijos: 20 & $22.7 \%$ \\
\hline & Uno: 21 & $23.9 \%$ \\
\hline & Dos: 35 & $39.8 \%$ \\
\hline & Tres: 12 & $13.6 \%$ \\
\hline
\end{tabular}


Fstudio psicológico como base del estudio de la diversidad humana

continua Cuadro 1

Caracteristicas

- Edad de los hijos

- Personal doméstico

- Tipo de vivienda

- Segunda vivienda

- Vehículo propio

\section{Distribucion}

Sin hijos: 20

Pequeños (0-5): 18

Medianos (6-14): 30

Mayores $(\geq 15): 20$

$2.7 \%$

$20.4 \%$

$34.09 \%$

$22.7 \%$

Sin personal doméstico: $29 \quad 33.0 \%$

Externa alterna: 35

$39.8 \%$

Externa diaria: 18

$20.5 \%$

Interna: 6

$6.8 \%$

$81.8 \%$

$9.1 \%$

$8.0 \%$

Chalet independiente:

$77.3 \%$

Sin segunsa vivienda: 68

$22.7 \%$

Con segunda vivienda: 20

$5.7 \%$

Sin vehículo: 5

Con vehículo: 83

El análisis de los datos descriptivos confirma que los participantes utilizados reflejan sociológicamente los estratos a los que se pretende que pertenezcan, de acuerdo con la descripción de la sociedad española (de Miguel, 1996).

\section{Instrumentos}

Encuesta de datos demográficos.- La selección de datos demográficos recogidos está basada en la bibliografía ya citada sobre las grandes encuestas de distribución de tiempo realizạdas. Su análisis a lo largo de este trabajo ha servido sobre todo para asegurarnos de que el grupo de sujetos utilizado no difería sociológicamente de su grupo de pertenencia teórico. 
Autorregistro de Distribución del Tiempo (ADIT).- Los instrumentos tradicionales para evaluar la distribución del tiempo se basan sobre todo en. entrevistas a los sujetos, e, incluso, en el caso de parejas, la estimación se hace a través de entrevistas sólo a la esposa. La influencia del recuerdo y de la subjetividad son evidentes en estos casos. Nos basamos en los trabajos sobre lo que se denomina diario de tiempo, time diary). La revisión de Harvey (1990) incluye estudios sobre la confiabilidad y validez básicas de estos tipos de instrumentos. La creación del instrumento (Sánchez-López, Quiroga, Sánchez-Herrero y Doblas, 1992) se ha basado en los siguientes puntos:

- Revisión de los instrumentos anteriores

- Discusión en grupo (incluyendo estudiantes postgraduados enrolados en un curso de especialización)

- Aplicación mediante estudio piloto a profesores/as de la Facultad de psicología de la UCM, a los que se pedía su valoración en todos los aspectos (incluidos los formales).

- El resultado es el instrumento que se presenta en el Anexo A.

\section{Procedimiento}

1. Normas de aplicación (centradas en conseguir una buena motivación de los sujetos y la comprensión de la tarea). Además de las normas por escrito, con detalladas explicaciones de cómo rellenar los instrumentos, se realizaba con cada pareja:

- Entrevista previa de motivación y explicación detallada

- Seguimiento telefónico

- Garantía de anonimato

- Delimitación de la semana estándar de la vida de la pareja

2. Transformación de los datos para ser manejados: dada la cantidad de datos disponibles, estos eran difícilmente manejables, por lo que se recurrió a la construcción de la hoja de tiempos y a la agrupación de actividades (Anexo 1). 


\section{Resultados}

Los análisis de datos realizados son los adecuados a las condiciones de los datos con respecto al nivel de medida de las variables utilizadas y al número de sujetos de cada grupo. Se ha calculado los estadísticos básicos para el análisis descriptivo de la muestra. Asimismo se ha utilizado clusters para la construcción de grupos, tablas de contingencia y análisis de varianza, cuando ha sido posible.

En primer lugar, se presenta los perfiles encontrados (G1, G2, G3) con la distribución del tiempo durante la semana, es decir, durante el tiempo laborable (TL), durante el fin de semana TFS) y la combinación del perfil de ambos tiempos (TL/FS). Todos estos resultados se presentan en los cuadros $2,3,4,5,6$ y 7 .

La caracterización de los grupos se resume en el Cuadro 8. Por último en los cuadros 9 y 10 se presentan las relaciones en distribución de tiempo $\mathrm{y}$ las variables demográficas.

\section{Cuadro 2}

Perfiles de estilo de vida (G1, G2, y G3) basado en tiempo laboral $(N=74)$

\begin{tabular}{|l|r|r|r|c|c|}
\hline Actividades & G1(38) & G2(26) & G3(10) & $\mathrm{T}$ & $\mathrm{P}$ \\
\hline \multirow{2}{*}{ domésticas } & 457.50 & 152.88 & 1600.50 & 82.27 & 0.000 \\
& 321.59 & 165.30 & 482.65 & & \\
comunes & 16.57 & 12.69 & 315.00 & 18.89 & 0.000 \\
& 55.67 & 38.11 & 381.77 & & \\
genéricas & 3469.74 & 3060.58 & 3220.50 & 11.09 & 0.000 \\
& 339.9 & 311.26 & 442.7 & & \\
hijos & 252.63 & 96.92 & 570.00 & 8.94 & 0.000 \\
& 324.23 & 135.248 & 484.25 & & \\
ocio & 926.05 & 848.08 & 1495.50 & 8.12 & 0.001 \\
& 469.82 & 336.43 & 407.68 & & \\
trabajo & 1993.02 & 2995.38 & 0.00 & 243.77 & 0.000 \\
& 383.525 & 403.6 & 0 & & \\
\hline
\end{tabular}




\section{Cuadro 3}

Caracterización psicologica de cada uno de los tres grupos (TL)

\begin{tabular}{|c|c|c|}
\hline G 1 & $\begin{array}{l}\text { medio doméstica } \\
\text { alto genérica } \\
\text { medio hijos } \\
\text { medio bajo ocio } \\
\text { medio trabajo }\end{array}$ & equilibrio \\
\hline G 2 & $\begin{array}{l}\text { bajo doméstica } \\
\text { bajo genética } \\
\text { bajo hijos } \\
\text { bajo ocio } \\
\text { alto trabajo }\end{array}$ & predominio trabajo \\
\hline G 3 & $\begin{array}{l}\text { alto hijos } \\
\text { alto doméstica } \\
\text { alto comunes }\end{array}$ & $\begin{array}{c}\text { predominio casa } \\
\text { (con hijos) } \\
\text { (amas de casa) }\end{array}$ \\
\hline
\end{tabular}

\section{Cuadro 4}

Perfiles de estilo de vida $(G 1, G 2$, y $G 3)$ basado en tiempo fin de semana $(N=74)$

\begin{tabular}{|l|r|r|r|r|l|}
\hline Acrivadides & G1 (3.8) & G2(26) & G3(10) & I & P \\
\hline domésticas & 56.91 & 185.13 & 115.46 & 11.36 & 0.000 \\
& 78.39 & 90.93 & 76.83 & & \\
comunes & 10.58 & 19.73 & 20.13 & 0.38 & 0.000 \\
& 23.57 & 53.86 & 35.48 & & \\
genéricas & 745.14 & 828.55 & 760.85 & 3.53 & 0.035 \\
& 106.56 & 97.07 & 107.32 & & \\
hijos & 176.03 & 104.21 & 21.51 & 14.64 & 0.000 \\
& 167.64 & 107.35 & 41.86 & & \\
ocio & 312.35 & 280.66 & 488.88 & 60.91 & 0.000 \\
& 66.97 & 82.55 & 75.91 & & \\
trabajo & 153.08 & 19.73 & 20.92 & 21.29 & 0.000 \\
& 127.95 & 42.44 & 49.11 & & \\
\hline
\end{tabular}


Estudio psicológico como base del estudio de la diversidad humana

\section{Cuadro 5}

Caracterización psicológica de cada uno de los tres grupos (PFS)

\begin{tabular}{|c|c|c|}
\hline G 1 & $\begin{array}{c}\text { bajo doméstica } \\
\text { alto hijos } \\
\text { medio ocio } \\
\text { alto trabajo }\end{array}$ & $\begin{array}{c}\text { función paterna }+ \\
\text { trabajo } \\
\text { (no dom.) }\end{array}$ \\
\hline G 2 & $\begin{array}{c}\text { alto doméstica } \\
\text { alto genérica } \\
\text { medio hijos } \\
\text { bajo ocio } \\
\text { bajo trabajo }\end{array}$ & $\begin{array}{c}\text { implicación } \\
\text { doméstica }\end{array}$ \\
\hline$G 3$ & alto ocio \\
muy bajo hijos & descanso \\
\hline
\end{tabular}

\section{Cuadro 6}

Perfil de estilo de tiempo laborable/ tiempo fin de semana (TL/FS)

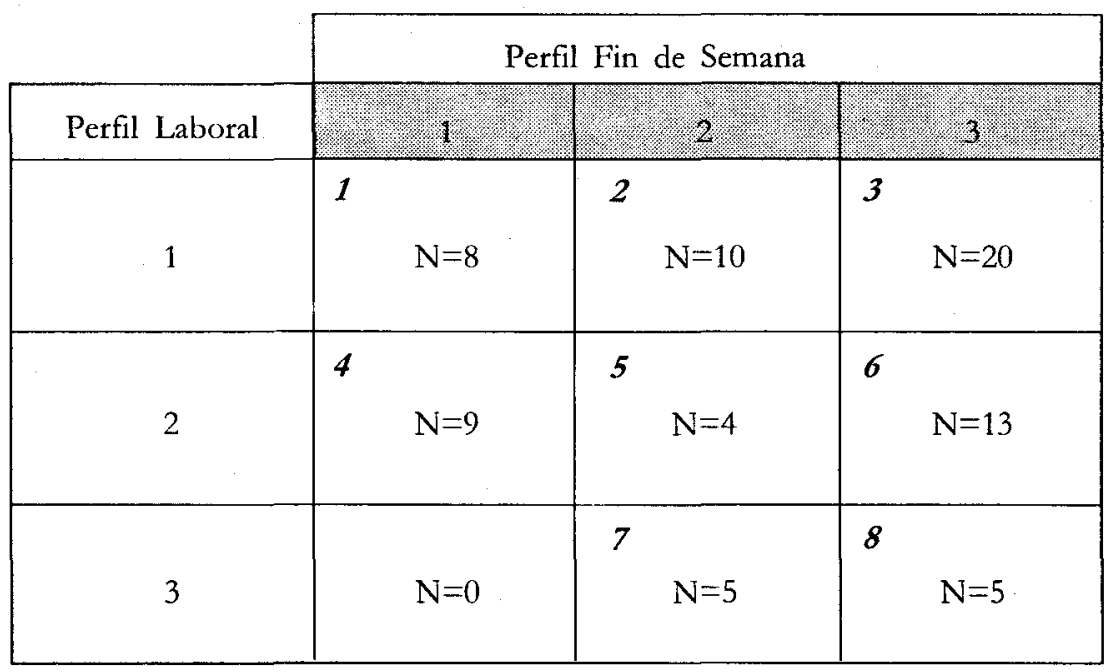




\section{Cuadro 7}

Caracterización psicológica de los grupos combinados (TL/FS)

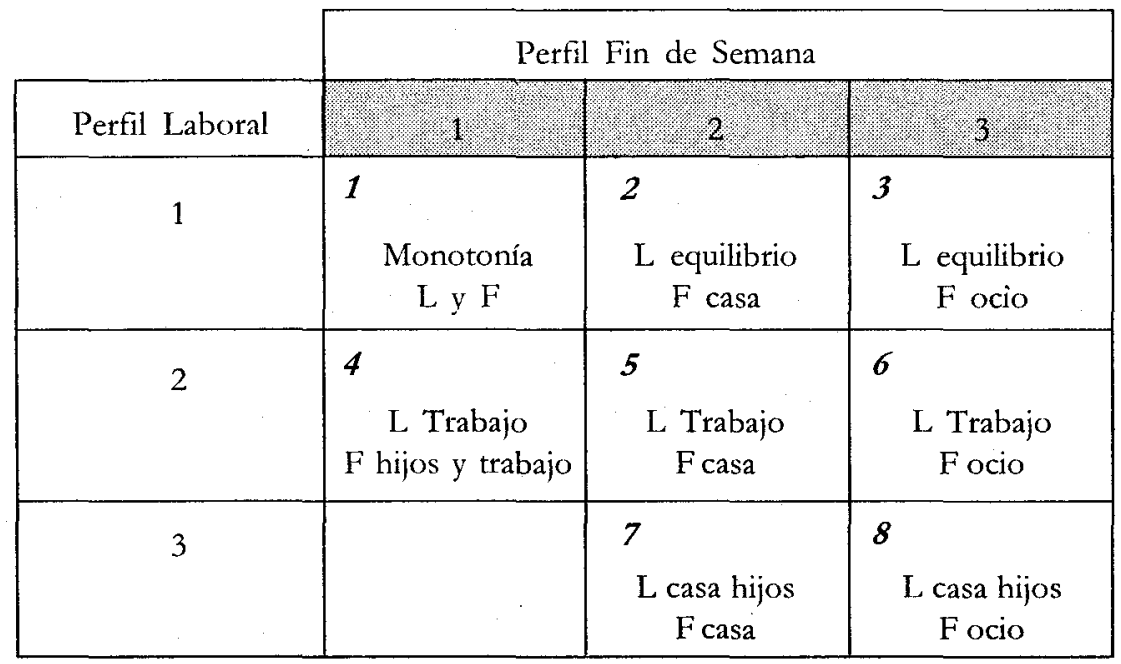

\section{Cuadro 8}

Resumen de la caracterización psicológica de todos los grupos

\begin{tabular}{|c|c|c|}
\hline Pcrfiles & Grupos & Caracterización \\
\hline Tiempo & 1 & equilibrio (doméstico, hijo, trabajo, ocio) \\
laboral (TL) & 3 & $\begin{array}{c}\text { predominio trabajo } \\
\text { predominio casa }\end{array}$ \\
\hline Tiempo fin & 1 & función paterna+trabajo (no doméstica) \\
semana (TFS) & 2 & implicación doméstica \\
& 3 & descanso \\
\hline Combinación & 1 & monotonía laborales y fin de semana \\
de & 2 & laborales, equilibrio, fin semana doméstica \\
perfiles de & 3 & laborales, equilibrio, fin semana, ocio \\
tiempo & 4 & laborales, trabajo, fin semana, hijos y trabaj. \\
(TL/FS) & 5 & laborales, trabajo, fin semana, casa (dom.) \\
& 6 & laborales, trabajo, fin semana, ocio \\
& 7 & laborales, casa, hijos, fin semana, casa \\
& 8 & laborales, casa, hijos, fin semana, ocio \\
\hline
\end{tabular}


Estudio psicologico como base del estudio de la divetsidad humana

\section{Cuadro 9}

Resumen de la relación entre perfil laboral y fin de semana y las variables demográficas

\begin{tabular}{|l|c|c|c|c|c|c|c|c|}
\cline { 2 - 9 } & \multicolumn{3}{c|}{ Variabales Dernograficas } \\
\cline { 2 - 9 } & \multicolumn{2}{c|}{ Sexo } & \multicolumn{2}{c|}{ T.Pareja } & \multicolumn{2}{c|}{ Edad } & \multicolumn{2}{|c|}{ Tener/no hijos } \\
\hline & $\mathrm{F}$ & $\mathrm{P}$ & $\mathrm{F}$ & $\mathrm{p}$ & $\mathrm{F}$ & $\mathrm{P}$ & $\mathrm{F}$ & $\mathrm{P}$ \\
\hline Perfil L/FS & 24.14 & 0.00001 & 32.27 & 0.000 & 0.582 & 0.56 & 1.37 & 0.51 \\
\hline Perfil. FS & 11.24 & 0.003 & 12.22 & 0.001 & 1.108 & 0.33 & 1.03 & 0.59 \\
\hline
\end{tabular}

\section{Cuadro 10}

Resumen de la relación entre perfil L/FS y variables demográficas

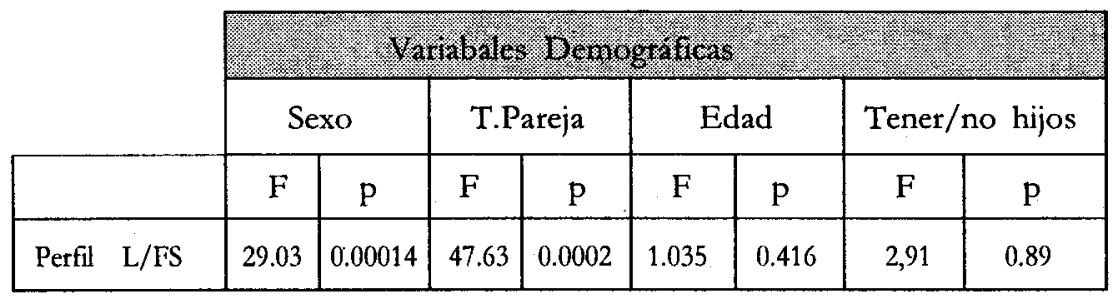

\section{Discusión}

Los datos confirman la hipótesis 1 y la hipótesis 2. Podemos utilizar razonablemente la distribución de tiempo como operativización del estilo de vida. Puesto que el propósito del presente trabajo sólo era comprobar que esto era asi, omitimos las relaciones específicas entre los distintos perfiles de distribución de tiempo y las variables demográficas, en aras de complicar excesivamente la exposición; no obstante, las relaciones aparecidas son enormemente interesantes, por lo que las publicaremos en 
un próximo trabajo. En resumen, el sexo se relaciona con estilos de vida seguramente debido a factores relacionados con el rol sexual.

Con respecto al tipo de pareja, es probable que cada tipo de pareja tenga sus propias recompensas, sus propias satisfacciones y también sus propios problemas y frustraciones por lo que parece lógico que el tipo de pareja (así como el sexo), module la distribución del tiempo que hace el sujeto.

La única variable demográfica que no se relaciona con los perfiles de ditribución de tiempo es la de ciclo vital. Quizás sería lógico esperar que tanto la edad de los sujetos como el hecho de tener/no tener hijos se relacionaran con estilo de vida. Para explicar este resultado podemos avanzar dos hipótesis: bien la distribución de esta variable en nuestros sujetos no es adecuada, y con otros sujetos, con otra distribución, aparecía esta relación, bien es que los sujeto "han hecho abstracción" de su edad y del hecho de tener o no hijos para organizar su tiempo. Para ser más exactos, quizás han hecho esta abstracción algunos sujetos, dependiendo de su sexo, por ejemplo, lo que explicaría la importancia de la variable sexo dentro de las variables demográficas.

Por último, a pesar de estas conclusiones positivas, no podemos pasar por alto el hecho de que, en cierto sentido, la utilización del estilo de vida que se ha hecho en este trabajo adolece de una cierta característica de "estructura". Al fin y al cabo, lo que se ha hecho es una "foto fija" de cómo el sujeto distribuye su tiempo en un momento determinado. Haria falta aplicar un método longitudinal para ver si hay un estilo a través del cambio, esas regularidades de segundo orden a las que hace referencia Larsen (1989). Pero también en otro sentido, hemos iniciado una línea de verdadero estilo"; cuando comparamos y combinamos el Perfil Tiempo laboral y el perfil tiempo fin de semana, hemos cambiado las circunstancias y hemos logrado una constancia, un estilo. En este sentido, los resultados pueden servir como punto de partida para ir profundizando en la conceptualización del estilo de vida a través de la distribución del tiempo. 


\section{Perspectivas futuras}

Resumidamente, éstas serian las principales perspectivas abiertas por los resultados de este trabajo:

1. Sería conveniente completar el análisis de las actividades realizado hasta ahora. En la bibliografía aparece el término "uso policrónico del tiempo" (Kautman, Lane y Lindsquist, 1991) para referirse a la combinación de actividades realizadas simultáneamente. Las instrucciones dadas a los sujetos permitían que éstos anotaran actividades simultáneas en el autorregistro, aunque hasta ahora sólo se ha contabilizado una en cada momento temporal, la principal. La propuesta, tomando como base la de los autores citados, sería la de evaluar si se producen diferencias en el uso policrónico frente al uso monocrónico del tiempo, si esas diferencias están relacionadas sólo con el tipo de actividades y el momento del día, o si, por el contrario, estarían relacionadas también con diferencias individuales y/o con la interacción entre sujeto/actividad/momento del día.

2. Con respecto a las variables demográficas:

- En primer lugar, habría que repetir el mismo análisis con respecto a las variables que constituyen el ciclo vital, para ver si se repiten los resultados o no (falta de relación con el estilo de vida) y poder escoger entre las dos alternativas de explicación propuestas.

- Habría que completar el análisis de la variable sexo con la variable género, es decir, incorporando al sexo biológico el sexo social, como las actitudes con respecto a los papeles sexuales. (Hay indicios en la bibliografía de que estas actitudes están relacionadas con el estilo de vida que el propio sexo).

3. Se deberia realizar el mismo análisis hecho hasta ahora después del paso del tiempo, para comprobar si existen o no las consistencia de segundo orden de las que habla Larsen (1989), con lo que podríamos estudiar el estilo como "patrón de cambio". 


\section{Referencias}

Argyle, M. (1987). The Psychology of happiness. London: Methuen and Co. Barling, J. (1990). Employment, stress and family functioning. Londres: John Wiley and Sons.

Buss, D.M. y Cantor, N. (1989). Personality Psycbology. Recents trends and emerging directions: Nueva York: Springer-Verlag.

Campbell, A., Converse, P.E. Y Rodgers, W.L. (1976). The Quality of American Life. Nueva York: Sage.

Carlson, R. (1971). Where is the person in personality research? Psycbological Bulletin, 75, 203-219.

Contrada, R.J., Wrigth, R.A. y Glass, D.C. (1985). Psychopsysiologics correlates of Type A behavior: Comments on Houston (1983) and Holmes (1983). Joumal of Research in Personality, 19, 12-30.

Cooper, K.J., Stern, H. y Mitchell, A. (1960). Consumer values and demand. Business Intelligence Program, SRI Internacional.

Cooper, K., Chassin, L., Braver, S., Zeiss, A. y Khavari, K.A. (1986). Correlates of mood and marital satisfaction among dual-worker couples. Social Psycbology Quarterly, 49, 4, 322-329.

Durán, $\mathrm{M}^{\mathrm{a}}$ A. (1987). De puertas adentro. Madrid: Instituto de la Mujer. Epstein, S. (1983). A research pardigm for the study $p$ f personality and emotions.

Nebraska Symposium on Motivation, 1982, 91-154.

Furnham, A. (1995). The relationship of Intelligence to Cognitive Learning Style and Achievement. En D.H. Saklofske, y M. Zeidner, International Handbook of Personality and Intelligence. Perspectives on Individual Diferences.

Nueva York: Plenum Press, 397-413.

Gilbert, L.A. (1985). Stress and Coping. En Gilbert, L.A. Men in dual career families: current realities and future prospects. Hillsdale: Lawrence Erlbaum. 91-117.

Harvey, A.S. (1990). The Measurement and analysis of Time Use. Social Indicators Research, 23, 4, 309-336.

Hendry, L., Shucksmith, J., Love, J. y Glendinning, A. (1993). Youg people's leisure and lifestyles. Nueva York: Routledge.

Ibañez, E. (1990). Psicologia de la salud y estilos de vida. Valencia: Promolibro. 
Izquierdo, J., Del Río, O. y Rodriguez, A. (1988). La desigualdad de las mujeres en el uso del tiempo: Madrid: Instituto de la Mujer.

Kautman, C.F., Lane, P.M. y Lindquist, J.D. (1991). Exploring more than 24 hours a day: A preliminary investigation at polichronic time use. Joumal of Consumer Research. 18, 392-401.

Krahé, B. (1990). Situation cognition and coherence in personality. An individualcentred approach. Cambridge University Press.

Larsen, R.J. (1989). A process approach to personality psychology: utilizing time as a facet of data. En Buss, D.M. y Cantor, N., Personality Psychology. Recent treds and emerging directions. Nueva York: SpringerVerlag, 177-193.

Larson, R.W., Richards, M.H., Raffaelli, M., Ham, M. y Lewell, L. (1990). Ecology of deppression in late childhood and early adolescence: A profile of daily states and activities. Joumal of Abnormal Psychology, 1, 92-102.

Leslie, L.A. y Anderson, E.A. (1988). Men's and women's participation in domestic roles: Impact on quality of life and marital adjustment. Joumal of Family Psychology, 2, 2, 212-226.

Marina, J.A. (1993). Teoría de la inteligencia creadora. Barcelona: Anagrama. McGrath, J. (1988). The Social Psychology of time. Guilford: Guilford Press. Miguel, A. de (1996). La sociedad española, 1995-96. Madrid: Editorial Complutense.

Quiroga, M.A. (1994). Los estilos cognitivos. En J. Sánchez-Cánovas y M.P. Sánchez-López, Psicologia Diferencial: Diversidad e Individualidad bumanas. Madrid: Centro de Estudios Ramón Areces, 317-375.

Quiroga, M.A. y Sánchez-López, M.P. (1987). Los estilos cognitivos: concepto y clasificación. Una revisión crítica. En M.P. SánchezLópez, M.P. y M.A. Quiroga, (eds.). Lecturas para las prácticas de Psicología Diferencial. Madrid: Facultad de Psicologia de la UCM. Quiroga, M.A. y Sánchez-López, M.P. (1997). Análisis de la insatisfacción familiar. Psicotbema. 9, 1, 69-82.

Radmika, S. y Prakash, I.J. (1987). Factors related to mental health of dualcareer couples. Journal of Personality and clinical studies, 3, 2, 131-134. 
Rapaport, R. Y Rapaport, R.N. (1969). "The dual carrer family: A variant pattern and social change". Human Relations, 22, 3-30.

Reuchlin, M. (1990). Différences individuelles dans le dévelopment conatif. Paris P.U.F.

Royce, J.R. y Buss, A.R. (1983). Theory of Personality and Individual Differences: Factors, Systems and Processes. Englewood Cliffs, N.J.: Prentice-Hall. Royce, J.R. y Powell, A. (1983). Teoría multifactorial sistemática. Exposición sucinta. Estudios de psicología, 4, 76-127.

Sánchez-Cánovas, J. y Sánchez-López, M.P. (1994). Psicología Diferencial: Diversidad e Individualidad bumanas. Madrid: Centro de Estudios Ramón Areces.

Sánchez-López, M.P. y Quiroga, M.A. (1995). Relaciones entre satisfacción familiar y Laboral: Variables moduladoras. Andes de Psicología, 11, 1, 65-78.

Sánchez-López, M.P. Quiroga, M.A., Sánchez-Herrero, S. y Doblas, T. (1992). El reparto de responsabilidades del bohar entre los miembros de la pareja en tres tipos de parejas. Informe de investigación. Instituto de la Mujer. Scase, R. Y Goffee, R. (1989). Reluctant managers. Their work and lifestyles. Londres: Unwin Hyman.

Sekaran, U. (1985). The paths to mental health: An exploratory study of husbands and wives in dual-career families. Joumal of Occupational Psychology, 58, 129-137.

Shulman, B.H. y Mosak, H.H. (1988). Manual for life style assessment. Muncie, IN: Accelerated Development Pub.

Strack, F., Argyle, M. y Schwartz, N. (1991). Subjective well-being: An interdisciplinary perspective. International Series in Experimental Social Psychology, 21. Pergamon Pres.

Witkin, H.A. (1949). The nature and importance of individual differences in perception. Journal of Personality, 18, 145-170.

Yogev, S. (1982). Happiness in dual-career couples: changing research, changing values. Sex Roles, 8, 6, 593-605. 


\section{Anexo A}

Autorregisro de Distribución del Teimpo (ADIT)

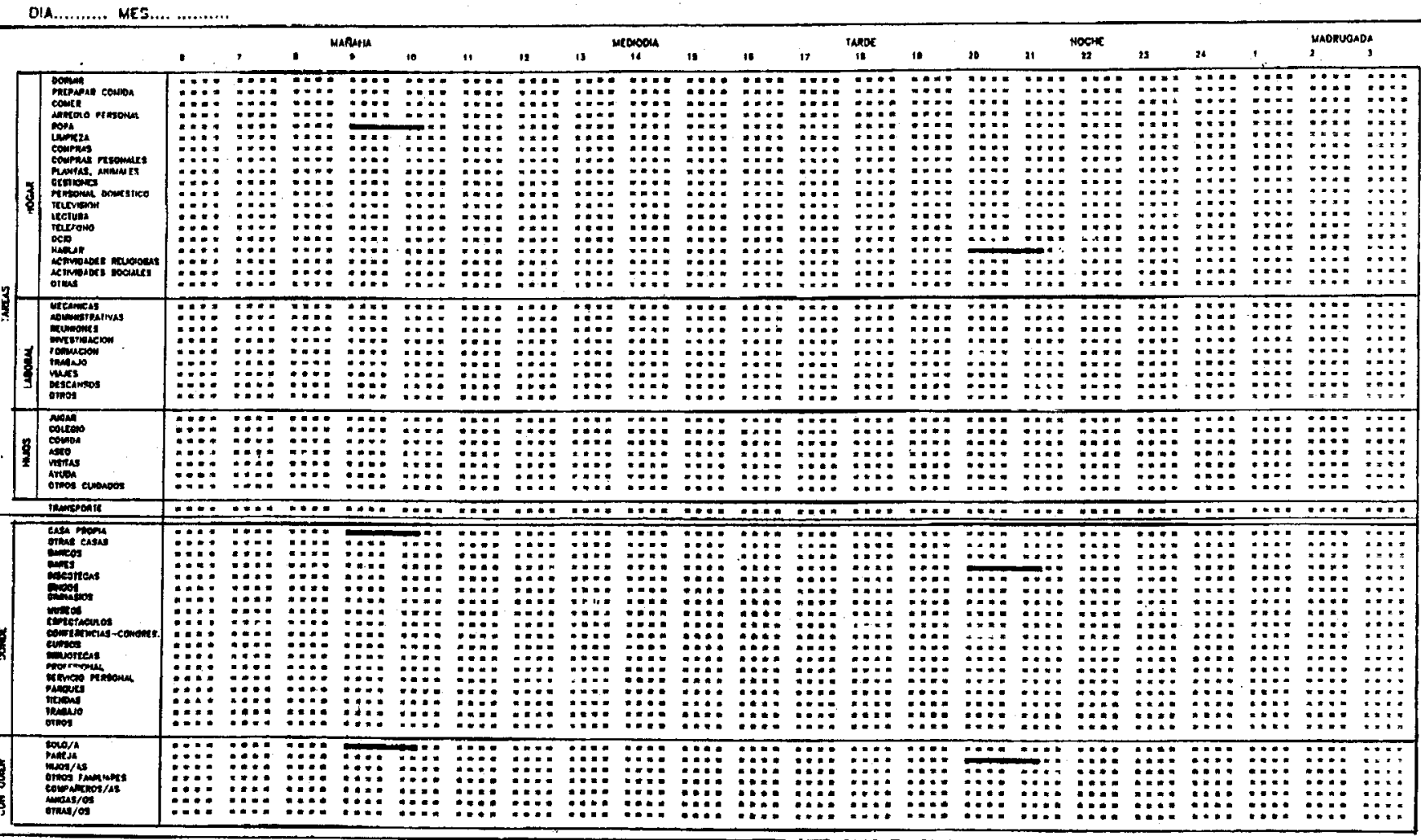


Construcción de los 6 grupos de actividades a partir del autorregistro de "Distribución del tiempo"

\section{ACTIVIDADES DOMÉSTICAS}

Se han sumado todas las actividades claramente domésticas como preparar comida, arreglar ropa, limpiar, las compras NO personales, y gestión.

\section{ACTIVIDADES DE OCIO}

Contiene la suma de lectura, ver televisión, ocio, compras personales. cenar con amigos, hablar y cuidar plantas y animales.

\section{ACTIVIDADES COMUNES}

Esta actividad está constituida por las actividades religiosas y las actividades sociales.

\section{ACTIVIDADES GENÉRICAS}

Bajo esta denominación se han unido todas las actividades que no cumplen los requisitos de las demás variables. Son ocupaciones generales que sirven para llevar a cabo las demás funciones. Incluye los transportes, dormir y comer (incluido Dormir fuera y Comer con Televisión y Otras).

\section{ACTIVIDADES RELACIONADAS CON EL TRABAJO}

También estas tareas tenían un apartado propio en el autorregistro, dividido en ayudar con las tareas del colegio, la comida, el aseo,ayuda en general, visitar, jugar y otras. 